\title{
Importancia de la formación en procesos de aplicación de política pública para emprendimientos solidarios: caso Medellín*
}

\author{
Importance of training in processes of the application of public policy \\ for joint ventures: the Medellin case
}

Importância da formação em processos de aplicação de política pública para empreendimentos solidários: caso Medellín

\section{Martha del Socorro Alzate Cárdenas ${ }^{1}$ Olga Lucía Arboleda Álvarez ${ }^{2}$}

Recibido: 6 de septiembre de 2018
Aceptado: 28 de mayo de 2019
Publicado: 21 de julio de 2019

Cómo citar este artículo: Alzate Cárdenas, M. S. y Arboleda Álvarez, O. I. (2019). Importancia de la formación en procesos de aplicación de política pública para emprendimientos solidarios: caso Medellín. Cooperativismo \& Desarrollo, 27(2), 1-15. doi: https://doi.org/10.16925/2382-4220.2019.02.08

* Artículo de investigación producto de dos proyectos de investigación desarrollados en los años 2015 y 2016 por el grupo de investigación ECOSOL de la Universidad Católica Luis Amigó, en alianza estratégica con el Observatorio de Planeación Local y Presupuesto Participativo de la Personería de Medellín y el grupo de investigación GICEA de la Corporación Universitaria Uniminuto, respectivamente. https://doi.org/10.16925/2382-4220.2019.02.08

1 Facultad de ciencias administrativas, económicas y contables Medellín, Colombia. Correo electrónico: martha.alzateca@amigo.edu.co. ORCID: https://orcid.org/0000-0001-5683-7238

2 Facultad de ciencias administrativas, económicas y contables Medellín, Colombia. ORCID: https://orcid.org/0000-0002-1436-904X 


\title{
Resumen
}

Este artículo realiza una reflexión sobre la importancia de la formación en procesos de aplicación de política pública para emprendimientos solidarios, a la luz de organizaciones democráticas, autogestionarias, humanitarias y sin ánimo de lucro. El proceso de construcción del artículo tuvo en cuenta aspectos de la política pública de economía solidaria iniciada en Medellín a partir del 2011 articulados a los procesos de formación. Se apoyó en algunos testimonios de beneficiarios, operadores y funcionarios de la administración municipal que han tenido una relación directa con dicha política pública, además de entrevistas con expertos del tema de algunos países de Latinoamérica. Las personas consultadas coinciden en la necesidad de replantear la formación, antes de aplicarse en otras regiones de Colombia.

Palabras clave: Formación, economía solidaria, política pública

\begin{abstract}
This article reflects on the importance of training in public policy application processes for solidarity projects, in the light of democratic, self-managed, humanitarian and non-profit organizations. The process of construction of the article, took into account aspects of the public policy of solidarity economy started in Medellin as of 2011 , articulated to the training processes and supported by some testimonies of beneficiaries, operators and officials of the municipal administration that have had a direct relationship with this public policy, in addition to interviews with experts on the subject of some countries in Latin America. The people consulted agree on the need to rethink training, before being applied in other regions of Colombia.
\end{abstract}

Keywords: Training, Solidarity Economy, Public Policy.

JEL classification: L31, L26, I31, M13, A2

\section{Resumo}

Palavras-chave: Este artigo realiza uma reflexão sobre a importância da formação em processos de aplicação de política pública para empreendimentos solidários, à luz de organizações democráticas, autogeridas, humanitárias e sem fins lucrativos. 0 processo de construção deste artigo considerou aspectos da política pública de economia solidária iniciada em Medellín, Colômbia, a partir de 2011, articulados aos processos de formação. Esteve apoiado em alguns depoimentos de beneficiários, operadores e funcionários da administração municipal que têm relação direta com essa política pública, além de entrevistas com especialistas do tema de alguns países da América Latina. As pessoas consultadas coincidem na necessidade de repensar a formação antes de ser aplicada em outras regiões da Colômbia.

Palavras-chave: economia solidária, formação, política pública.

\section{Introducción}

En la ciudad de Medellín, Colombia, se tienen avances significativos en el marco legal de políticas públicas que posibilitan la creación, fortalecimiento y consolidación de formas asociativas o emprendimientos solidarios. Esto se evidencia a través del Acuerdo 41 de 2011 (Concejo de Medellín 2011a), "Por medio del cual se adopta la política pública de economía social y solidaria en el Municipio" y su decreto reglamentario 486 (Alcaldía de Medellín 2015a). Este artículo realiza una reflexión sobre la importancia 
de la formación en procesos de aplicación de política pública para la economía social solidaria tomando como referencia algunos testimonios y entrevistas informales con expertos en el tema.

Se tomó el concepto de formación empresarial solidaria, como aquella que potencia procesos de transformación social, capacidades críticas y reflexivas de las personas, a la luz de organizaciones democráticas, humanitarias, autogestionarias, solidarias y sin ánimo de lucro, para el desarrollo de actividades productivas o de servicios.

La principal motivación para esta reflexión fue el hallazgo más relevante de los procesos investigativos, asociado con las falencias de formación derivadas de la aplicación de la política pública para la economía social solidaria en los últimos años en la ciudad de Medellín. Lo anterior se pudo evidenciar en los testimonios de las personas que hicieron parte de la memoria oral recogida mediante la técnica de grupos focales y operativizada con entrevista semiestructurada, aplicada a los actores de la política pública: beneficiarios, operadores y administración municipal, además de las entrevistas con expertos en el tema.

Vale la pena aclarar que esta reflexión también se inició como ponencia en el VII Congreso nacional de investigadores en economía solidaria, realizado en la ciudad de Pereira el día 21 de septiembre de 2016, en el eje temático: "Las políticas públicas y el desarrollo del país". Se espera que con esta reflexión se haga un aporte a las estrategias de formación propuestas en el sistema de economía solidaria en el país en los diferentes escenarios de formulación y aplicación de la política pública y economía social solidaria

\section{Reflexión desde las consideraciones teóricas}

Para el desarrollo de esta reflexión, se inició el análisis de la categoría "formación" en tres direcciones. La primera de ellas se asocia con los modelos de operación del programa "economía solidaria", contemplado en los dos últimos planes de desarrollo de Medellín, dicho análisis, está directamente ligado con el ecosistema de emprendimiento de Medellín. En éste se tiene en cuenta la cadena de valor en relación con sensibilización hacia el emprendimiento, identificación de ideas de negocio, formulación de planes de negocio, puesta en marcha de esos planes y aceleración (Alcaldía de Medellín 2015b). 
La segunda dirección considera la formación del grupo emprendedor o base asociativa, donde se privilegian los contenidos de un curso básico de economía solidaria, entre los cuales se incluyen, según la Unidad Administrativa Especial de Organizaciones Solidarias- Ministerio del Trabajo, República de Colombia (2013): cultura solidaria, historia de las organizaciones, principios, valores, tipos de organizaciones solidarias, formas de constitución, emprendimiento solidario y desarrollo territorial, entre otros.

La tercera dirección obedece a la reflexión realizada al interior del grupo de investigación, donde se toma el concepto de formación en economía solidaria desde un referente más amplio. Desde un referente transversal que también coincide con la aplicación de la política pública de economía solidaria en Brasil, Ecuador, México, Uruguay, Argentina y Colombia, específicamente Medellín, y que conduce a hablar en términos de educación en economía solidaria, pedagogía de la solidaridad y formación empresarial solidaria, desde los cuales básicamente deben promoverse los principios y valores como la democracia, la participación y la igualdad. Con ello se busca también aportar a procesos autogestionarios organizacionales y comunitarios, en el marco de políticas de desarrollo sostenible y de mejoramiento de las condiciones y la calidad de vida de los asociados y de las comunidades, lo cual se torna coherente y tiene sus antecedentes desde lo dispuesto por el Departamento Administrativo Nacional de la Economía Solidaria (DANSOCIAL), en el presente, Unidad Administrativa Especial de Organizaciones Solidarias, y el Ministerio de Educación Nacional, que mediante la directiva 031 del año 2000, determinaron el marco conceptual de la educación solidaria, dando orientaciones para su adecuada promoción e implementación y definiéndola como:

El proceso permanente orientado a fortalecer la práctica de valores de solidaridad, cooperación y ayuda mutua, con el fin de generar actitudes y conductas que permitan consolidar un compromiso social frente a la misión que deben cumplir las Organizaciones Solidarias de conformidad con las previsiones de la Ley 454/98.

La educación es entendida como un instrumento que permite asegurar la multiplicación de la teoría y los métodos organizativos y formar a los asociados y dirigentes en las especificidades que caracterizan las prácticas propias de las empresas de economía solidaria. 
Al respecto son válidas las palabras del maestro Antonio Fabra Ribas, citado en Arboleda y Zabala (2005, p. 33) cuando dice: "la educación cooperativa no se propone fabricar sabios, sino hacer hombres en toda la acepción de la palabra: capacitados física y moralmente para saber a cada momento lo que deben hacer y como tienen que hacerlo."

Según Salgado (2015) este concepto de educación en economía solidaria implica colocarse en la vía de generar procesos de trasformación económico y social y construir el saber desde las prácticas que las empresas solidarias han desarrollado en cada territorio y localidad. Sin embargo, el tener un referente de base teórica pone de manifiesto que el desarrollo de los procesos de trasformación pasa por la interpretación y la actualización de las necesidades de las comunidades y de la población.

Por ello, el componente educativo es un pensamiento que debate sobre los fines de la economía y de las intenciones en la construcción del Estado. La educación solidaria deberá pretender la promoción de "[...] una corriente de pensamiento sobre una economía alternativa, pensada no solo desde el mercado o desde el Estado, sino fundamentalmente pensada desde el ámbito de la cultura y desde la ética de la responsabilidad compartida para un desarrollo humano sustentable y autosostenido en una sociedad solidaria" (Martínez 1995, citado en Salgado 2015).

El componente de educación para la economía solidaria es pues, "un eje dinamizador de comunidades con deseo de cambio, en un contexto concreto" (Moreno, 1995, p. 186), lo cual permite afirmar que la educación solidaria debe tener una mirada para el desarrollo en el contexto local.

Este concepto de educación en economía solidaria se presenta estrechamente ligado al de pedagogía solidaria, y en este sentido la reflexión debe iniciarse por la pregunta acerca de la pedagogía. Esta se concibe como la reflexión sobre los procesos educativos, como la disciplina que estudia la educación y la enseñanza y que se ocupa de trazar la teoría sobre la educación. Así, la pedagogía puede definirse como "el conjunto de saberes, conocimientos, principios, estrategias y prácticas históricamente sistematizadas, organizadas y contextualizadas, que permiten orientar los procesos de formación y desarrollo humano integral de las personas, por medio de acciones pertinentes y contextualizadas" (Funlam, 2012, p. 76).

En ese orden de ideas se dirá que la pedagogía de la solidaridad es aquella que se manifieste en términos contraventores frente al orden mecanicista y que eduque para la libertad. En dicha pedagogía: 
El proceso de enseñanza - aprendizaje conlleva la urgencia de proveer capacidades críticas y reflexivas fundamentadas, que posibiliten realizar procesos de desaprendizaje, pero también de nuevas construcciones cognitivas, todo ello mediado por procesos de reflexividad, en la perspectiva de hacer de cada quien una mejor persona, que da lo mejor de sí, para su propio bien y el de los demás [...] la educación solidaria, educa para ese fin, como un todo integrado, orientada por un marco filosófico e ideológico que distingue y fortalece la identidad doctrinaria del movimiento cooperativo y del sector solidario. (Arboleda y Lopera, 2011, p. 58).

En consonancia con estos referentes de educación y pedagogía para la solidaridad, el concepto de formación, del cual se partió, considera un ser humano que está en condiciones de continuo mejoramiento y que es capaz de proyectarse y trascender, y donde la formación sería entonces, a la manera de Gadamer (1997, p. 43) "el proceso por el que se adquiere cultura: el modo específicamente humano de dar forma a las disposiciones y capacidades naturales de los seres humanos". Resultan pertinentes también los aportes de Mariluz Restrepo (en línea) cuando dice: "el concepto de formación va más allá del mero cultivo de capacidades previas. En la formación uno apropia por entero aquello en lo cual y a través de lo cual uno se forma. La formación no trata de cuestiones de procedimiento o de comportamiento, sino del ser en cuanto ente devenido; su principal característica es ese mantenerse abierto hacia lo otro, hacia puntos de vista distintos y más generales".

Específicamente sobre el concepto de formación para el modelo cooperativo y solidario, pueden asumirse las precisiones que realiza Silva (2010, p. 173), quien ubica dicha formación como un ámbito de la educación, al respecto dice:

No basta con entregar técnicas y conocimientos a los asociados. Esto de por si es vital e importante para el desarrollo empresarial, pero no es suficiente. Además de tener asociados, directivos y funcionarios bien entrenados, también es necesario contar con seres humanos sensibles, capaces de ofrecer soluciones a las demás personas, capaces de impactar favorablemente en la sociedad y de generar mecanismos eficientes de cooperación sin dejar a un lado la necesidad de crecer y sostenerse económicamente. Las cooperativas son empresas de personas y por tanto la formación debe ser complemento indisoluble de la educación, la capacitación, la asistencia técnica y la investigación. 
A partir de estas consideraciones, la educación como formación identifica, según Salgado (2015), varios aspectos a tener en cuenta en la perspectiva de configurar la educación para la economía solidaria desde elementos de formación integral que contribuyan al desarrollo humano de las comunidades:

- $\quad$ Necesidad de una teoría propia, la cual puede sustentar de manera más sólida una lógica y una racionalidad basada en los factores de comunidad y trabajo contemplados como fundamentales en la teoría económica comprensiva del profesor Razeto (1988).

- Necesidad de proyectos de mediano y largo plazo promovidos por organizaciones solidarias y por universidades, ya que los principios de la educación cooperativa expresados en la ley cooperativa en Colombia no pueden ser el referente único y no son suficientes para construir un sistema de educación solidaria.

- El desarrollo local basado en el diagnóstico de necesidades de las organizaciones solidarias, asumido como bitácora hacia la construcción de programas curriculares.

- Replica de las buenas prácticas que se han dado en las apuestas educativas de la economía solidaria, como referentes para la generación de procesos de apropiación del conocimiento, que redunden en el bienestar de la comunidad y no como un conocimiento indexado.

- Establecimiento de relaciones con movimientos sociales de la economía solidaria, que permitan conocer otras formas de expresión de la economía social y solidaria y ayuden a generar nuevos procesos innovadores de gestión comunitaria.

Ahora bien, es imprescindible asumir el concepto de formación en economía solidaria en articulación con lo empresarial, toda vez que el mismo Concejo de Medellín (2011b) medianteel acuerdo 55 de 2011, establecela Política Municipal de Desarrollo Empresarial para la ciudad de Medellín, en el marco del Plan Estratégico de Emprendimiento Regional, cuya finalidad está en directa relación con la promoción del "empleo de calidad" o "trabajo decente" a "partir del apoyo y el fomento a las prácticas de emprendimiento y empresarismo que generen dinámica, competitividad y proyección económica para la ciudad con el fin de construir tejido empresarial". Además, el artículo 5 de este acuerdo define emprendimientos de inclusión socioeconómica como: 
Emprendimientos con niveles medios o bajos de innovación que responden a necesidades específicas del contexto local —barrios, comunas y corregimientos - y de la población que se beneficia del mismo, los cuales buscan trascender de la economía popular a la economía hacia las iniciativas asociativas, sociales y solidarias, con mayores niveles de sostenibilidad, que ha futuro generan trabajo decente. Son generadores de riqueza familiar.

De aquí que finalmente esta reflexión, asume la categoría de formación como "formación empresarial solidaria", desde la generación de procesos de transformación social, potenciando capacidades críticas y reflexivas a la luz de una organización solidaria que sea capaz de "asociarse basada en la solidaridad, para trabajar por el beneficio de los asociados o de la sociedad en general [desarrollando una actividad productiva o de servicios]", como lo establece la Unidad Administrativa especial de Organizaciones Solidarias-Ministerio del Trabajo: República de Colombia (2013, p. 28) "Emprendimiento solidario", hacia la conformación de una "Empresa solidaria".

De esta manera la formación empresarial solidario busca que las personas "[piensen, sientan y actúen creativamente], [...] hacia la generación de bienestar social, calidad de vida y valor para [cada] individuo y su comunidad que les permite desarrollar proyectos socio económicos en actuación cooperativa y en relación con el entorno". Visto así el emprendimiento es "la disposición de un grupo de personas para aportar sus habilidades en la creación de nuevas ideas y proyectos de carácter colectivo, para el bienestar económico y social de los integrantes del grupo o de la comunidad en general, a través de la gestión adecuada y autónoma de los recursos necesarios".

Por tanto, la formación empresarial solidaria generará como resultados la creación, fortalecimiento y consolidación de empresas solidarias como formas de organización de recursos materiales y humanos, para satisfacer necesidades y lograr beneficios sociales por medio de un objetivo económico, de acuerdo a una forma diferente de hacer economía y de entender el desarrollo (Unidad Administrativa especial de Organizaciones solidarias- Ministerio del Trabajo, 2013).

Al articular entonces la formación empresarial solidaria con la cadena de valor de emprendimiento surgen las siguientes etapas propias del ecosistema de emprendimiento. (Alcaldía de Medellín, 2015a).

- Sensibilización.

- Identificación de oportunidades de negocio.

- Formulación del plan de negocio. 
- Puesta en marcha.

- Aceleración.

En las tres primeras etapas, que corresponden a sensibilización, identificación y formulación, se encuentran los emprendedores potenciales, que son aquellos que tienen una iniciativa socioempresarial y quieren desarrollarla. En la cuarta etapa, que es la de puesta en marcha, se reconocen los emprendedores nacientes, empresarios nuevos, que son los que iniciaron la aventura de gestionar una empresa solidaria. Finalmente, en la quinta etapa o de aceleración se encuentran los empresarios establecidos, que se caracterizan por tener tiempo y experiencia en la gestión de la empresa solidaria (Unidad Administrativa especial de Organizaciones solidarias-Ministerio del trabajo, 2013, p. 28).

\section{Reflexión desde los principales testimonios, con respecto a la formación}

En este apartado se realizó una selección de aspectos de la formación, a partir de testimonios de actores del proceso para Medellín y expertos en el tema de algunos países de Latinoamérica.

Brasil: sobresalen eventos académicos para difusión del conocimiento como foros, en ellos se trata de fortalecer las empresas del sector con la partición en redes de producción, comercialización y consumo, así como las redes de suministro (Nagao, 2016).

Ecuador: el Instituto de Economía popular y Solidaria (IEPS), ha sido muy representativo para este país en los procesos de formación en educación solidaria. Allí se ha desarrollado un sistema de información estructurado y definido para la toma de decisiones (Sánchez, 2016).

México: en este país se han desarrollado redes universitarias, con el fin de impulsar los procesos de formación y cultura de la cooperación en articulación con la ecología. (Rojas, 2016).

Uruguay: predomina la iniciativa de la Universidad de la República, con la que se originó el Plan Montevideo para la economía social y solidaria. Este plan cuenta con el aval del Departamento de Desarrollo e integración regional para hacer la articulación con la industria, turismo y producción del agro (Marti, 2016). 
Argentina: se dan procesos colaborativos entre la universidad, la empresa representado en instituciones que apoyan al sector cooperativo y mutualista- y el Estado (Muglia, 2016).

Colombia: en el ámbito nacional se desarrollan programas y proyectos por parte de instituciones públicas y privadas para investigación del sector y divulgación de resultados, así como para procesos de pedagogía solidaria. Generalmente esos procesos de desarrollan por organismos de segundo de grado del sector solidario. La ley 1780 de 2016 "por la cual se incentiva el empleo juvenil", establece la obligación de "impartir educación cooperativa y solidario en todos los niveles educativos del país", con lo cual se han dinamizado los procesos de formación de la economía solidaria (Zabala, 2016).

Para el caso de Medellín, se escucharon algunas críticas a los procesos de formación empresarial solidaria (Salgado, et al., 2015), en aspectos relacionados con demasiada capacitación, rapidez en la presentación de los contenidos, información muy repetitiva y que no llega al público objetivo de la intervención. Esto indica que será necesaria la revisión de los cronogramas, tiempos de intervención y contenidos de la capacitación de tal manera que tengan una relación directa con las lógicas de formación de acuerdo con la cadena de valor del emprendimiento social solidario.

Sin desconocer que la tarea educativa y formativa debe ser "una tarea para las escuelas y en general para la educación formal y no formal" (Gonzales, 2001, p. 9), pero también, de todos los actores involucrados en el proceso. Hay que advertir que se requieren propuestas de mediano y largo plazo que permitan asumir los retos educativos que las practicas socioeconómicas de la economía solidaria demandan, formaciones que vayan más allá de los simples cursos básicos ordenados por la ley, aplicados a través de proyectos que sean asumidos y desarrollados por organizaciones solidarias y por instituciones de la educación superior debidamente acreditadas. Tal como lo señala Álvarez, citado por Silva (2010, p. 99):

[...] se requiere un compromiso claro e inaplazable por parte de los comités de educación para hacer de la educación un proceso de aprendizaje no solo dirigido a las personas, sino también a la organización; de manera que la educación pueda inducir cambios en los patrones de vida de las personas, en las conductas organizacionales y genere espacios donde la cooperación y la confianza sean parte del quehacer racional humano, a pesar de nuestros connaturales errores. 
Con respecto a la formación en economía solidaria, también se encontraron en Medellín, inconformidades derivadas de aspectos como: formación en economía solidaria con sesgos en la explicación de formas jurídicas y trabajo en equipo, curso básico de economía solidaria para cumplir con el requisito, formación en economía solidaria solo desde la forma jurídica de la organización, falta de credibilidad en la economía solidaria, casos de capacitación en economía solidaria donde se hace mucho énfasis a odiar el capitalismo. Estas posturas hacen pensar la formación en economía solidaria como una propuesta alternativa, no sustituta del sistema capitalista que, sustentada en valores como la solidaridad, la ayuda mutua y la cooperación, permite la expresión y el desarrollo de diversas experiencias alternativas a las hegemónicas.

De todas maneras, los testimonios de los beneficiarios de la Política Pública de Economía Social Solidaria en Medellín, reflejan la necesidad de hacer una revisión minuciosa de estos procesos en el país. De tal manera que la aplicación de dicha política verdaderamente fomente procesos de transformación social, con prácticas democráticas, humanitarias, autogestionarias y solidarias que potencien capacidades críticas y reflexivas a la luz de un emprendimiento social y solidario. Un beneficiario de una política pública debe comprender e interiorizar lo que significa el trabajo solidario como factor determinante del éxito en este tipo de emprendimientos y por ende en la construcción de comunidad como categoría dominante (Torres y Santander, 2013).

Lo anterior hace necesario pensar en procesos de formación en coherencia con los principios de la economía solidaria, teniendo presente el fortalecimiento del ser solidario - pensar, sentir hacer economía solidaria- Situación que también la perciben operadores de la política al manifestar la necesidad de planear capacitaciones que trasciendan más en un pensamiento colectivo que individual (Salgado, et al, 2015). Es necesario trabajar por la construcción colectiva de una cultura de la solidaridad desde la aplicación de la política pública.

De otro lado, esta reflexión toma en cuenta algunos testimonios que le dan sentido y significado a favor de los procesos de formación en economía solidaria que coinciden con los emprendimientos sociales y solidarios exitosos, lo que deja entrever que ellos, los emprendimientos sociales y solidarios exitosos, están conformados por grupos de personas que se asocian alrededor de la actividad económica que soporta el emprendimiento solidario, conservan motivación para unir sus esfuerzos para llevar adelante ese ideal, a la vez que obtienen un reconocimiento por parte de la comunidad. También evidencian prácticas solidarias y humanistas al interior de ellos. La investigación pudo evidenciar la existencia de casos tan importantes, donde la formación ha permitido una sensibilización empresarial hacia el oficio, potenciar la capacidad de servicio y la capacidad de asombro hacia el medio y de hacer las cosas bien hechas. 


\section{Conclusiones}

Al revisar los referentes en las conceptualizaciones expuestas y leyendo a la luz de ellas, los sentidos y significados que provienen de la interpretación realizada al testimonio de algunos beneficiarios de la Pública de Economía Solidaria en la ciudad de Medellín, si bien algunos integrantes de los emprendimientos solidarios, ven la formación como una fortaleza para el desarrollo de sus actividades empresariales y han participado en procesos de formación, estos procesos aún están distantes del propósito de instalar un pensamiento solidario para la administración de empresas de este tipo, para la creación de circuitos económicos, para la conformación de redes y para el fortalecimiento de un sector solidario que propenda por las acciones estratégicas del desarrollo solidario. Reflexión que debe hacerse en todos los ámbitos del país.

Así aunque en Medellín, los beneficiarios del programa "Economía solidaria", valoran la economía solidaria como alternativa a las formas tradicionales de empresa, y plantean que la política pública sea efectiva para que la visibilización del modelo solidario no solo sea una reacción sino una realidad, es necesario reflexionar sobre la necesidad de hacerse sentir como un modelo que propone una nueva economía local, al desarrollar productos y servicios que puedan estar en la mismas condiciones que las empresas individuales y que puedan negociar con el Estado.

La formación para el emprendimiento solidario no debe ser distante en calidad, de la formación dada por el ecosistema del emprendimiento individual, formar empresarios con las características sociales y solidarias, requiere potenciar cambios y mejoramientos, si no en todos los beneficiarios, sí en la mayoría de ellos, en sus familias y las comunidades a las que pertenecen, de tal manera que se identifiquen con la creación, fortalecimiento y consolidación de unidades productivas asociativas mediante el acceso a conocimientos útiles para el inicio o desarrollo de los emprendimientos.

Igualmente, la formación debe garantizar la sensibilización y acercamiento al tema de la economía solidaria, comprendiendo la importancia de sus principios y haciendo conciencia de que a través de sus prácticas lo que busca lograrse es el bien común. Se debe alcanzar a determinar el avance en la creación, fortalecimiento y consolidación de las empresas asociativas, de tal manera que se relacione lo aprendido con las exigencias en la configuración empresarial y en el fortalecimiento del grupo, hasta el punto que los beneficiarios se conviertan en referentes de la economía local desde sus barrios. 


\section{Referencias}

Alcaldía de Medellín. (2015a). Decreto 0486 de 2015, por medio del cual se reglamenta el Acuerdo 41 de 2011 que adopta la política pública para la economía social solidaria en el Municipio de Medellín. Gaceta oficial, 4298. Recuperado de: https://www.medellin.gov.co/normograma/docs/a_conmed_0041_2011.htm

Alcaldía de Medellín. (2015b). Radicado 201500437306 comunicación de Coordinación de Economía Solidaria a Personería de Medellín. Medellín, Colombia.

Arboleda Álvarez, O. L. y Zabala Salazar, H. (2005). La economía solidaria en Antioquia. Estado del arte 1960 -2003. Medellín, Colombia: Fondo Editorial Funlam.

Arboleda Álvarez, O. L. y Lopera García, L. D. (2011). Concepciones y prácticas sobre pedagogía y educación cooperativa y solidaria. Desicio, 29, 56-61. Recuperado de: https://cdn.crefal.org/ CREFAL/revistas-decisio/decisio29_saber9.pdf

Concejo de Medellín. (2011a). Acuerdo 41. Política pública para la economía social y solidaria en el Municipio de Medellín. Gaceta oficial, 3968, 48-53. Recuperado de: http://www.culturaemedellin.gov.co/sites/CulturaE/ciudade/Documents/Acuerdo\%2041\%20de\%202011\%20 Empresas\%20Base\%20social\%20y\%20Solidaria.pdf

Concejo de Medellín. (2011b). Acuerdo 55, por el cual se establece la política municipal de desarrollo empresarial para la ciudad de Medellín, en el marco del plan estratégico de emprendimiento regional. Gaceta oficial, 3977, noviembre 30. Recuperado de: http://www. culturaemedellin.gov.co/sites/CulturaE/ciudade/Documents/Acuerdo\%2055\%20de\%20 2011\%20Pol\%C3\%ADtica\%20de\%20Desarrollo\%20Empresarial.pdf

Congreso de la República de Colombia. (1998). Ley 454 de 1998, por medio de la cual se Ley 454 de 1998, por la cual se determina el marco conceptual que regula la economía solidaria, se transforma el Departamento Administrativo Nacional de Cooperativas en el Departamento Nacional de la Economía Solidaria, se crea la Superintendencia de la Economía Solidaria, se crea el Fondo de Garantías para las Cooperativas Financieras y de Ahorro y Crédito, se dictan normas sobre la actividad financiera de las entidades de naturaleza cooperativa y se expiden otras disposiciones. Congreso de Colombia. Bogotá, Colombia. 
Congreso de la República de Colombia. (2016). Ley 1780 de 2016, por medio de la cual se promueve el empleo y el emprendimiento juvenil, se generan medidas para superar barreras de acceso al mercado de trabajo y se dictan otras disposiciones. Recuperado de: http://es.presidencia. gov.co/normativa/normativa/LEY\%201780\%20DEL\%2002\%20DE\%20MAYO\%20DE\%20 2016.pdf

Fundación Universitaria Luis Amigó (Funlam). (2012). Proyecto Educativo Institucional-PEl. Medellín, Colombia: Fondo Editorial Funlam. Recuperado de: https://www.funlam.edu.co/modules/ documentosjuridicos/item.php?itemid=684

Gadamer, G. (1997). Verdad y Método I. Salamanca, España: Sígueme. Recuperado de: https:// www.iberlibro.com/Verdad-M\%C3\%A9todo-Tomo-II-Hans-Georg/5704806395/bd

Gonzáles Butrón, M. A. (2001). Economía social para la vida. Desafíos a la educación. Desicio, 29, 3-9.

Marti, J. P. (2016). Entrevista de audio. Sao Paulo. Recuperado de: https://www.youtube.com/ channel/UCP391YRAjSOdM_bwievgaZA

.Moreno Avendaño, J. del C. (1995). Historia y filosofía de la economía solidaria. Medellín, Colombia: Funlam.

Muglia, L. (2016). Entrevista de audio. Sao Paulo.

Nagao Menesez. (2016). Entrevista de audio. Sao Pablo.

Razeto Migliaro, L. (1988). Fundamentos de una teoría económica comprensiva. Santiago: Ediciones PET. Recuperado de: http://www.luisrazeto.net/content/fundamentos-de-unateor\%C3\%ADa-econ\%C3\%B3mica-comprensiva

Restrepo, M. (s.f.). El Currículo. Revista Al tema del Hombre. Recuperado de: http://www.chasque. net/frontpage/relacion/0703/curriculo.htm

Rojas, J. J. (2016). Entrevista de audio. Medellín, Colombia.

Sánchez, J. (2016). Institucionalidad y políticas para la economía popular y solidaria: balance de la experiencia ecuatoriana. En H. Jácome Estrella, J. Sánchez, J. Oleas, et al. Economía Solidaria Historias y prácticas de su fortalecimiento: Serie de estudios sobre Economía popular y Solidaria. Superintendencia de Economía Popular y Solidaria. 
Salgado Cañaveral, O. A. (2015). Educación solidaria: un instrumento para la transformación económica y social. Campus Fundación Universitaria Católica del Norte.

Salgado Cañaveral, O., Arboleda Álvarez, O. L., Alzate Cárdenas, M., et al. (2015). Política pública de economía solidaria en el contexto de planeación local y presupuesto participativo de Medellín 2008-2015, evaluación de efectividad. Medellín, Colombia: Artes y Letras.

Silva Díaz, J. A (2010). Educación para la cooperación. Bogotá, Colombia: Fondo Nacional Universitario.I.A.C

Torres Melo, J. y Santander A. J. (2013). Introducción a las políticas públicas: Conceptos y Herramientas desde la relación entre el Estado y Ciudadanía. Procuraduría General de la Nación. Bogotá, Colombia: IEMP Ediciones. Recuperado de: https://es.slideshare.net/ caritovillegas/introduccion-a-las-politicas-publicas

Unidad Administrativa Especial de Organizaciones Solidarias. Ministerio del trabajo y Ministerio de Educación. República de Colombia. (2000). Directiva 031 de julio 7 de 2000. Recuperado de: http://cooinpe.com/descargas/dansocial/DIRECTIVA\%20031\%20DE\%20 2000.pdf

Unidad Administrativa Especial de Organizaciones Solidarias. Ministerio del trabajo. República de Colombia. (2013). Cursos básico, medio y avanzado de Economía Solidaria. Recuperado de: http://www.orgsolidarias.gov.co/?q=educaci\%C3\%B3n-solidaria/herramientas-educati vas/herramientas-virtuales/curso-de-econom\%C3\%ADa-solidaria

Zabala, H. (2016). Entrevista audio. Medellín. 